\title{
BIOCHEMICAL, PURIFICATION, SEQUENCING AND ALIGNMENT STUDIES OF THE NOVEL POLYPHENOL OXIDASE ISOFORMS FROM MUSA ACUMINATA FRUIT PULP
}

\author{
N. Sajjad ${ }^{1}$, S. M. S. Naqvi ${ }^{1}$, M. J. Asad ${ }^{1}$, N. I. Raja ${ }^{2}$ M. Pusztai-Carey ${ }^{3}$ and M. S. Ahmad ${ }^{1 *}$ \\ ${ }^{1}$ University Institute of Biochemistry and Biotechnology (UIBB), ${ }^{2}$ Department of Botany, Pir Mehr Ali Shah, Arid \\ Agriculture University, Rawalpindi, Pakistan, \\ ${ }^{3}$ Department of Biochemistry, School of Medicine, Case Western Reserve University, OH, USA \\ *Corresponding Author's E-mail: dr.sheeraz@uaar.edu.pk
}

\begin{abstract}
Polyphenol oxidases (PPOs) are copper containing enzymes that play a significant role in the browning of fruits and vegetables by catalyzing hydroxylation and oxidation reactions. Currently, PPO gained significant interest of the researchers due to its potential applications in food, paper, pulp and, textile industries and also in pharmaceuticals. The present study was designed to purify and characterized PPO isoforms from fresh banana fruit pulp. The three novel active PPO isoforms $(65 \mathrm{kDa}, 45 \mathrm{kDa}, 28 \mathrm{kDa})$ were detected by gel filtration chromatography and sodium dodecyl sulfate polyacrylamide gel electrophoresis (SDS-PAGE). The purified isoforms were further studied for the optimum $\mathrm{pH}$ (6.5), temperature $\left(40^{\circ} \mathrm{C}\right)$, Michaelis constant $\left(\mathrm{K}_{\mathrm{m}}\right)$ and maximum reaction velocity $\left(\mathrm{V}_{\max }\right)$. The N-terminal microsequencing was performed on applied biosystem's pulse liquid protein sequencer and was found to be $28 \mathrm{kDa}$ (Alanine, proline, asparagine, serine, arginine) and $45 \mathrm{kDa}$ (Alanine, proline, isoleucine, alanine, proline). Sequences were aligned by CLUSTALW and showed significant similarity with previously reported banana PPOs.
\end{abstract}

Key words: PPO isoforms, gel filtration chromatography, N-terminal sequencing, multiple sequence alignment. https://doi.org/10.36899/JAPS.2021.2.0243 Published online October 03,2020

\section{INTRODUCTION}

Polyphenol oxidases (PPOs) are found in animals, plants, fungi and bacteria (Mayer et al. 2006). Tyrosinase, catechol oxidase and laccases are the three types of PPO which catalyze hydroxylation of monophenols to diphenols (cresolase activity) and oxidation of diphenols to quinone (catecholase activity) that further polymerized to brown pigments called melanins (Simsek et al. 2007; Queiroz et al. 2008). PPO enzyme has been studied in many fruits and vegetables due to its significant role in browning process (Wuyts et al. 2006; Anaya-Esparza et al. 2017; Jesus et al. 2018). Different studies have clearly shown that PPO enzyme is mostly involved in the enzymatic browning process of plant food that occurs during storage and processing (Munoa-pina et al. 2018). Researchers have found significant applications of PPO in various industries. In food industry PPO have wide range of applications as in the improvements in flavor of tea, coffee and cocoa, determination of ascorbic acid, pectin gelation of sugar beet and as a biosensor (Polaina and MacCabe, 2007). Recent research states that PPO has a significant role in increasing plant immunity (Taranto et al. 2017). PPO have potential applications in drug industries, biosensor and also in the degradation of phenolic compounds. PPO has been used in the synthesis of 3,4-dihydroxy-Lphenylalanine called L-DOPA which is used in the treatment of Parkinson's disease. PPO from fungi and potato play an important role in degradation of the organic contaminants (Hou et al. 2011) and in the formation of pigments (Mohammadi et al. 2002; Bravo and Osorio; 2016). It is also implicated in oxygen scavenging (Constabel et al. 2000). Tyrosinase is found generally in humans and animals. In skin, hair as well as in eye pigmentation it is mainly involved in melanin synthesis. Exoskeleton formation (Halaouli et al. 2006) is one of the most important functions of PPO in insects. Tyrosinase has a key role in the healing system of wounds (Kong et al. 2000). In case of any cut and damage to the plant, PPO oxidizes some phenolic compounds and form polymeric structure for the plant protection against the microorganisms and insects. In vegetables and fruits this process results due to enzymatic browning that cause significant loss to the food industries. Laccase, a type of PPO has a significant function in the lignification processes and it is also capable of cross linking the phenolic monomers. PPO plays an important role in lignin degradation in fungi. In fungi PPO is also involved in the detoxification process of toxic compounds, spore formation as well as pigmentation. The best studied role of PPO in bacteria is the melanins formation that provides protection to the spores of bacteria and cells against oxidants, UV radiations and free radicals (Claus and Decker 2006). In most recent studies PPO has been studied in many plant food such as mung bean sprouts (Sikora and Świeca, 2018), soursop nectar (Anaya-Esparza et al. 2017), acai 
fruit (Jesus et al. 2018) bayberry juice (Cao et al. 2017), blueberry (Siddiq and Dolan, 2017), tea leaf (Teng et al. 2017), and cape gooseberry (Bravo and Osorio, 2016).

Banana is considered a significant crop in the whole world and one of the most important food sources in the developing world (Paul et al. 2001). Currently commercialization of banana is increasing and expanding but during handling, storage and processing its tissues turn dark brown because of the PPO action (Sojo et al. 1998a). Fruits and vegetables are rich source of polyphenol oxidase enzyme. In previous studies, the molecular weight of purified PPO from banana (Musa sapientum) pulp was estimated to be 41000 and 42000 and the maximum PPO activity was found at $\mathrm{pH} 6.5$ and $30^{\circ} \mathrm{C}$ temperature and PPO was stable for 30 minutes above that temperature (Yang et al. 2000). In another study, PPO from one banana variety [Musa (AAA Group) 'Gros Michel'] was purified using Sephacryl S-200 HR and fast protein liquid chromatography on Mono Q column and PPO activity was maximum at $\mathrm{pH} 7$ (Chaisakdanugull and Theerakulkait, 2009). It was found that PPO forms four active bands with native PAGE and five active bands with SDS-PAGE and PPOs activity was optimum at $\mathrm{pH} 7$ (Karakus et al. 2009). The two active PPO isozymes were obtained from banana using sephadex column chromatography (Nematopour et al. 2008).

The main significant problem that confronts us after reviewing the literature about PPO purification from banana was that there was no detailed previous research available regarding banana (Musa acuminata, Cavendish subgroup of the AAA group). Only few studies have focused on PPO purification from other banana varieties. It was our interest to know whether the banana fruit pulp hold PPO isoforms or not.

Keeping in view the significant increase in PPOs importance in various fields and richness of PPO in banana, the present study was designed. The aim of this study is to explore the PPO enzyme in detail from fresh banana (Musa acuminata, Cavendish subgroup of the AAA group) fruit. We have extracted, purified and studied the biochemical properties of PPO. N-terminal sequencing of the purified isoforms was carried out for the first time and sequences were aligned. Phylogenetic tree was also constructed.

\section{MATERIALS AND METHODS}

Materials: Sodium phosphate buffer was used in the extraction. The protein precipitation was performed by centrifugation using cold acetone. Water 1525 binary pump with superdex column was used for high performance liquid chromatography (HPLC). Column chromatography with sephadex G-100 column was performed. NuPAGE Novex Bis-Tris gel in X Cell Sure Lock Mini Cell (Invitrogen, Thermo Fisher Scientific, cat no. NP0004) was used. Western blotting (wet blotting) was used for transferring the gel on polyvinylidene diflouride membrane (PVDF). The N-terminal microsequencing was performed using applied biosystem's pulse liquid protein sequencer (ABI Model 477A).

Extraction of polyphenol oxidase: The extraction procedure was used as explained by Mahmood et al. (2009) with some modifications. Fresh banana fruits were peeled and cut in small slices and crude extract for PPO extraction was prepared by homogenizing $80 \mathrm{~g}$ of sliced fruit in $100 \mathrm{~mL}, 0.1 \mathrm{M}$ sodium phosphate buffer $(\mathrm{pH} 7)$ using waring blender for $\sim 5 \mathrm{~min}$. Homogenate was centrifuged at $8000 \mathrm{rpm}$ for $\sim 90 \mathrm{~min}$. Supernatant was collected and stored at $4^{\circ} \mathrm{C}$ for further analysis.

\section{Purification of polyphenol oxidase}

Acetone precipitation of protein: The method was used as described by Guray (2009) with few modifications. Crude extract of fresh banana fruit pulp was subjected to acetone precipitation. Cold acetone (1.5 volumes) was added to crude extract (1 volume) and kept at constant stirring for 8 "h" at $4{ }^{\circ} \mathrm{C}$. Precipitates were collected from the extract after centrifugation at $8000 \mathrm{rpm}$ for $\sim 30 \mathrm{~min}$ and were dissolved in $10 \mathrm{~mL}$ of buffer $(0.1 \mathrm{M}$ phosphate, $\mathrm{pH} 7$ ).

Gel filtration chromatography: PPOs after acetone precipitation from the crude extract of banana were purified by high performance liquid chromatography. In order to get standard chromatogram, bovine serum albumin was initially injected into the HPLC superdex column, and then the purified sample $(1 \mathrm{~mL})$ after acetone precipitation was injected. Phosphate buffer, 0.1 $\mathrm{M}(\mathrm{pH}$ 7) was used for washing the column and each fraction $(3 \mathrm{~mL}$ volume) was collected during the complete running cycle ( $\sim 24 \mathrm{~min})$ of the sample. All the fractions were tested for the protein concentration and PPO activity. PPO assay was performed with catechol substrate. Fractions having PPO activity were stored at $4^{\circ} \mathrm{C}$ for further experiments (Karakus and Pekyardimci, 2009; Guven et al. 2017).

PPO was also purified by column chromatography with sephadex G-100 column $(1.2 \times 70$ $\mathrm{cm})$. Crude extract of fresh banana fruit pulp $(20 \mathrm{~mL})$ after acetone precipitation was loaded into the sephadex G-100 column and each fraction with $3 \mathrm{~mL}$ volume was collected after washing the column using buffer $(0.1 \mathrm{M}$ phosphate buffer, $\mathrm{pH}$ 7). All the fractions were obtained at the time until no absorbance was observed at $280 \mathrm{~nm}$. Protein concentration and PPO activity was determined from all the fractions. PPO active fractions were stored at $4^{\circ} \mathrm{C}$ and used in for further analysis (Guray, 2009).

Sodium dodecyl sulfate polyacrylamide gel electrophoresis (SDS-PAGE): It was performed by 
NuPAGEBis-Tris Mini Gels in X cell Sure Lock minicell using standard protocol. NuPAGE LDS sample buffer and NuPAGE reducing agent were added to prepare the samples $(100 \mu \mathrm{L})$. Heating $(5-10 \mathrm{~min})$ was done before loading the samples into the gel cassette wells. The $20 \mathrm{X}$ NuPAGE MES or MOPS sodium dodecyl sulphate running buffer $(50 \mathrm{~mL})$ was added to deionized water $(950 \mathrm{~mL})$ to prepare $1 \mathrm{X}$ Sodium dodecyl sulphate running buffer for the gel. Gel cassette was placed into the $\mathrm{X}$ cell sure lock and buffer chambers (upper and lower) were loaded with $1 \mathrm{X}$ running buffer. Gel was stained with staining solution (deionized water $55 \mathrm{~mL}$, methanol $20 \mathrm{~mL}$, stainer A $20 \mathrm{~mL}$, stainer B $5 \mathrm{~mL}$ ) after the completion of electrophoresis process $(200 \mathrm{~V}, 35$ $\min )$.

Measurement of PPO activity: PPO assay was done using the method Kumar et al. (2008) with little modifications. PPO activity was performed by taking 200 $\mathrm{mM}$ substrate solution and $0.1 \mathrm{M}$ phosphate buffer and the reaction rate was determined after the addition of enzyme solution. Catechol was used as a substrate. Activity for the enzyme was expressed in a unit that was defined as the amount of enzyme that causes 0.001 absorbance changes in $\sim 1 \mathrm{~min}$. Absorbance was taken using spectrophotometer (UV-Visible spectrophotometer Evolution 60) at $420 \mathrm{~nm}$.

\section{Studies of biochemical properties of PPO}

Optimum pH: The optimum PPO activity of purified banana PPO isoforms from fresh banana fruit pulp was determined using buffer with varying $\mathrm{pH}$ i.e. 3, 3.5, 4, $4.5,5,5.5,6,6.5,7,7.5$ and 8. Polyphenol oxidase assay was performed to observe the maximum activity of the enzyme by using catechol as substrate.

Studies of the optimum temperature: Enzyme solution was incubated at varying temperatures i.e. $30^{\circ} \mathrm{C}, 40^{\circ} \mathrm{C}$, $50^{\circ} \mathrm{C}, 60^{\circ} \mathrm{C}$, and $70^{\circ} \mathrm{C}$ for $15 \mathrm{~min}$ and the reaction rate was studied at room temperature by PPO assay (Guray, 2009).

Studies of the metal ions: Metal ions play an important part in the catalytic activity of many enzymes. In order to check the effect of metal ions on PPO activity of banana, metal ions such as $\mathrm{CuSO}_{4}, \mathrm{KCl}$ and $\mathrm{CaCl}_{2}(1 \mathrm{mM})$ were added into enzyme solution (Guray, 2009) and the reaction rate was determined by performing PPO enzyme assay using the above described method in the measurement of PPO activity.

Enzyme kinetics studies: Michael-Menten constant $\left(\mathrm{K}_{\mathrm{m}}\right)$ and maximum rate of the reaction $\left(\mathrm{V}_{\max }\right)$ for banana PPOs were determined. $\mathrm{K}_{\mathrm{m}}$ was calculated by Lineweaver-burk plot.

Protein microsequencing: Wet blotting was used to transfer the proteins from the gel to the
Polyvinylidenedifluoride (PVDF) membrane. Blotting buffers, PVDF membrane, whatman $3 \mathrm{MM}$ filter paper, blotting membrane, electroblotting equipment, electrophoresed gel, blotting cassette and sponge pad were used. The anode side of the blotting cassette was placed in a dish containing blotting buffer and a sponge pad was placed on the top of blotting cassette. The whatman $3 \mathrm{MM}$ filter paper (two pieces) was placed on the sponge pad and air bubbles were removed. Methanol was used to wet the PVDF membrane and was placed in the blotting buffer above the blotting sandwich. The SDS-PAGE gel was placed above the PVDF membrane. Another sponge pad was placed into the buffer above the gel and the cathode side of the blotting cassette was immersed on the sponge pad and fixed to the anode side. At the end the cassette was removed from the dish of buffer and was placed into the blotting tank filled with transfer buffer. It was connected to the power supply for two hours at $500 \mathrm{~mA}$. The $0.2 \%(\mathrm{w} / \mathrm{v})$ coomassie brilliant blue staining solution was used to stain the membrane for $2 \mathrm{~min}$ and then it was destained and washed with deionized water. Further the membrane was placed in a clean plastic bag for future use and N-terminal microsequencing was performed on applied biosystem's pulse liquid protein sequencer by Edman chemistry and Applied Biosystem's computer program.

Multiple sequence alignment and phylogenetic studies. Sequences were aligned in order to study the phylogenetic relationship of the purified PPO isoforms from fresh banana fruit pulp with previously reported PPO sequences from banana. Sequences were obtained (https://www.ncbi.nlm.nih.gov/) and converted to FASTA format and pasted into ClustalW (http://www.genome.jp/tools-bin/clustalw) for multiple sequence alignment.

Statistical analysis. All the experiments were performed in triplicates and data was analyzed by means \pm standard error (SE) using descriptive statistics and regression. Prism pad statistical software (GraphPad Prism, Version 5.02) was used in the preparation of graphs for studying the biochemical properties of the enzyme.

\section{RESULTS}

Preparation of Musa acuminata crude extract. Crude extract of banana was prepared and PPO assay was performed to determine PPO activity using catechol substrate. PPO activity from the crude extract of fresh banana fruit pulp was determined to be $5.59 \mu \mathrm{M} / \mathrm{min}$ and protein concentration was observed as $8.375 \mathrm{mg} / \mathrm{mL}$ (Table 1). 


\section{Purification of polyphenol oxidase}

Acetone precipitation. PPO activity after acetone precipitation was found to be $2.86 \mu \mathrm{M} / \mathrm{min}$ and protein concentration was taken as $3.844 \mathrm{mg} / \mathrm{mL}$ (Table 1).

Gel filtration chromatography. Fractions were tested for the PPO activity using catechol substrate and the PPO activity was observed in the fractions collected between 10-14.45 min (Fig 1). PPO purification with sephadex G100 column was performed and the PPO activity was observed between 17-23 fractions (Fig 2). Table 1 shows stepwise purification result of polyphenol oxidases from banana fruit pulp. Total PPO activity after gel filtration was taken as $1.698 \mu \mathrm{M} / \mathrm{min}$. Total protein concentration was determined to be $0.635 \mathrm{mg} / \mathrm{mL}$. Specific activity of PPOs was observed as $2.67 \mathrm{U} / \mathrm{mg}$. Purification folds and yield was calculated to be 4.04 and $30 \%$.

SDS-PAGE. Electrophoretic pattern of the gel indicates three isoforms of PPO (65 kDa, $45 \mathrm{kDa}, 28 \mathrm{kDa})$ from fresh banana fruit pulp (Fig 3).

Biochemical properties of banana PPO. PPO isoforms from fresh banana fruit pulp purified through HPLC were used for further biochemical properties analysis.

Determination of optimum pH. PPO activity was determined using $0.1 \mathrm{M}$ buffer (sodium phosphate) with varying $\mathrm{pH}(3,3.5,4,4.5,5,5.5,6,6.5,7,7.5$, and 8) during enzyme assay with catechol substrate. Absorbance was taken at $420 \mathrm{~nm}$ and the result showed that banana fruit pulp PPO activity was maximum at $\mathrm{pH} 6.5$ and 7 (Fig 4).

Determination of optimum temperature. The banana fruit pulp PPO isoforms was optimum at 30 to $40^{\circ} \mathrm{C}$ and above that temperature PPO activity was slightly less and starts decreasing (Fig 5).

Effect of metal ions on PPO activity. The varying concentration of metal ions was added in order to check the effect of metal ions on polyphenol oxidase activity of fresh banana fruit pulp. PPO assay for the metal ions was performed and it was found that metal ions neither increased nor decreased the PPO activity; copper ions were the only exception because PPO is the enzyme with dicopper center that's why the presence of copper ions (1mM Conc.) increased the PPO activity (Fig 6).

PPO kinetic studies. $\mathrm{K}_{\mathrm{m}}$ and $\mathrm{V}_{\max }$ values using catechol substrate under optimal conditions were determined by means of Lineweaver-burk plot and $\mathrm{K}_{\mathrm{m}}$ and $\mathrm{V}_{\max }$ for banana PPO $(53.33 \mathrm{mM}, 9.15 \mu \mathrm{M} / \mathrm{min})$ were calculated (Fig 7).

N-terminal microsequencing. N-terminal microsequencing was performed after transferring the gel to the PVDF membrane on an applied biosystem's pulse liquid protein sequencer. Edman chemistry was used with applied biosystem' $\mathrm{s}$ computer program for protein sequencing. N-terminal microsequence of the two purified isoforms from banana fruit pulp showed alanine, proline, asparagine, serine, arginine (28 kDa) and Alanine, proline, isoleucine, alanine, proline (45 $\mathrm{kDa})$ amino acid sequence (Fig 8a-e, Fig 9a-e).

Multiple sequence alignment and phylogenetic analysis. Sequences were aligned by CLUSTALW (Fig $10 \mathrm{a}$ and $\mathrm{b}$ ) and phylogenetic tree was drawned. It was found that PPO isoforms $(45 \mathrm{kDa}, 28 \mathrm{KDa})$ purified from banana fruit pulp in the given study have strong sequence similarity with the previously reported PPOs from other banana varieties (Accession no AHH92811.1 \& ACJ65307.1). The phylogenetic tree (Fig 11) showed two PPO isoforms B3 (28 kDa) and B4 (45 kDa) and its closed relation with AHH92811.1 \& ACJ65307.1 banana PPOs. These (AHH92811.1 \& ACJ65307.1) are tyrosinase isoforms and our purified isoforms have strong resemblance with tyrosinase, a type of PPO (Figs 12 a and b). PPO contain dinuclear copper center, three domains are usually present which include chloroplast transit peptide present at amino-terminal position and carboxyl-terminal position (Ono et al. 2006). The copper region $\mathrm{CuA}$ and $\mathrm{CuB}$ is present in central position (Fig 13). Three histidine residues help in functioning of each copper atom and form the active site (Ono et al. 2006).

Table 1. Stepwise Purification Result of Banana PPO

\begin{tabular}{llllll}
\hline Steps & $\begin{array}{l}\text { Total Activity } \\
(\boldsymbol{\mu M} / \mathbf{m i n})\end{array}$ & $\begin{array}{l}\text { Total Protein } \\
(\mathbf{m g} / \mathbf{m L})\end{array}$ & $\begin{array}{l}\text { Specific } \\
\text { Activity }(\mathbf{U} / \mathbf{m g})\end{array}$ & Yield (\%) & $\begin{array}{l}\text { Purification } \\
\text { (Folds) }\end{array}$ \\
\hline $\begin{array}{l}\text { Crude Extract } \\
\text { Acetone }\end{array}$ & 5.59 & 8.375 & 0.66 & 100 & 1 \\
Precipitation & 2.86 & 3.844 & 0.74 & 51 & 1.12 \\
Gel Filtration & 1.698 & 0.635 & 2.67 & 30 & 4.04
\end{tabular}




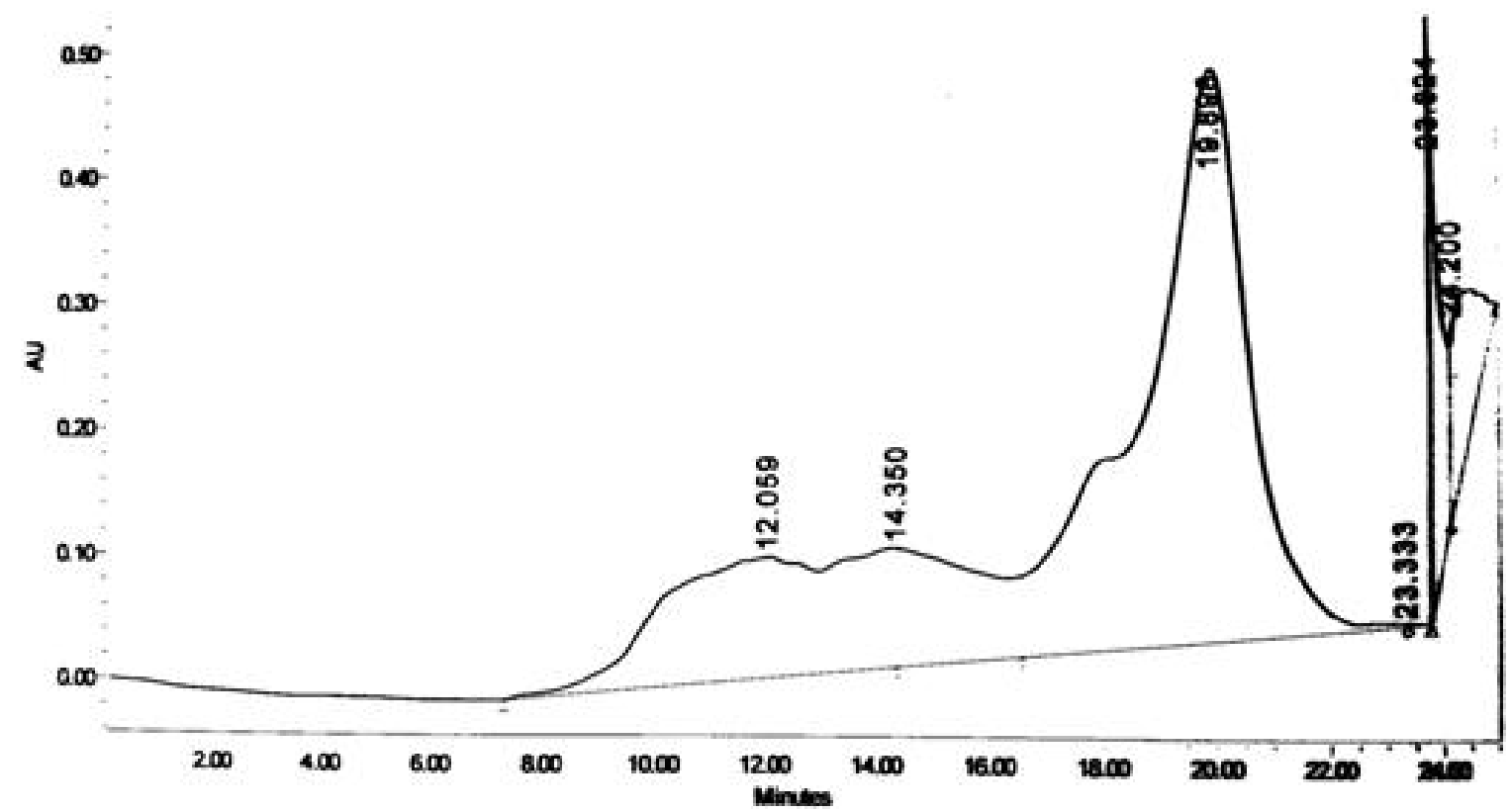

Figure 1. Elution profile of banana fruit pulp polyphenol oxidase isoforms obtained from high performance liquid chromatography using superdex column, retention time (RT) 10-14.35 minutes with 0.15 absorbance on spectrophotometer. Time period of 24 minutes is showing on horizontal axis and absorbance is showing on vertical axis. HPLC graph is showing different peaks at 10-24 minutes. There are elution of low molecular weight compounds present in the crude extract that are eluted later on around 24 min peak and afterwards.

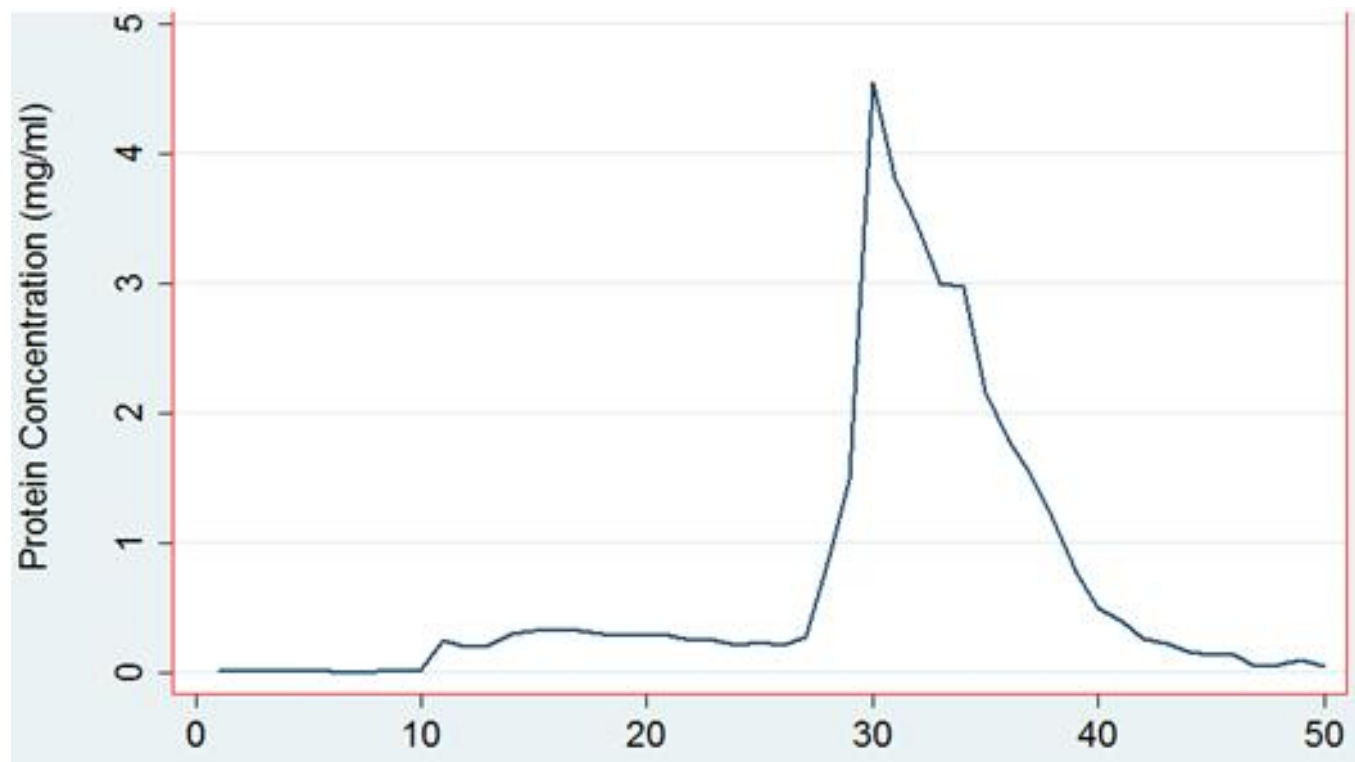

Fractions Obtained Through Elutions ( $3 \mathrm{ml} /$ fraction)

Figure 2. Column chromatography (Sephadex G-100) of banana fruit pulp PPO isoforms from crude extract after acetone precipitation. Polyphenol oxidase activity was observed between 17-23 fractions. Number of total fractions is shown on horizontal axis and protein concentration $(\mathrm{mg} / \mathrm{mL})$ on vertical axis. Total 50 fractions were collected. 


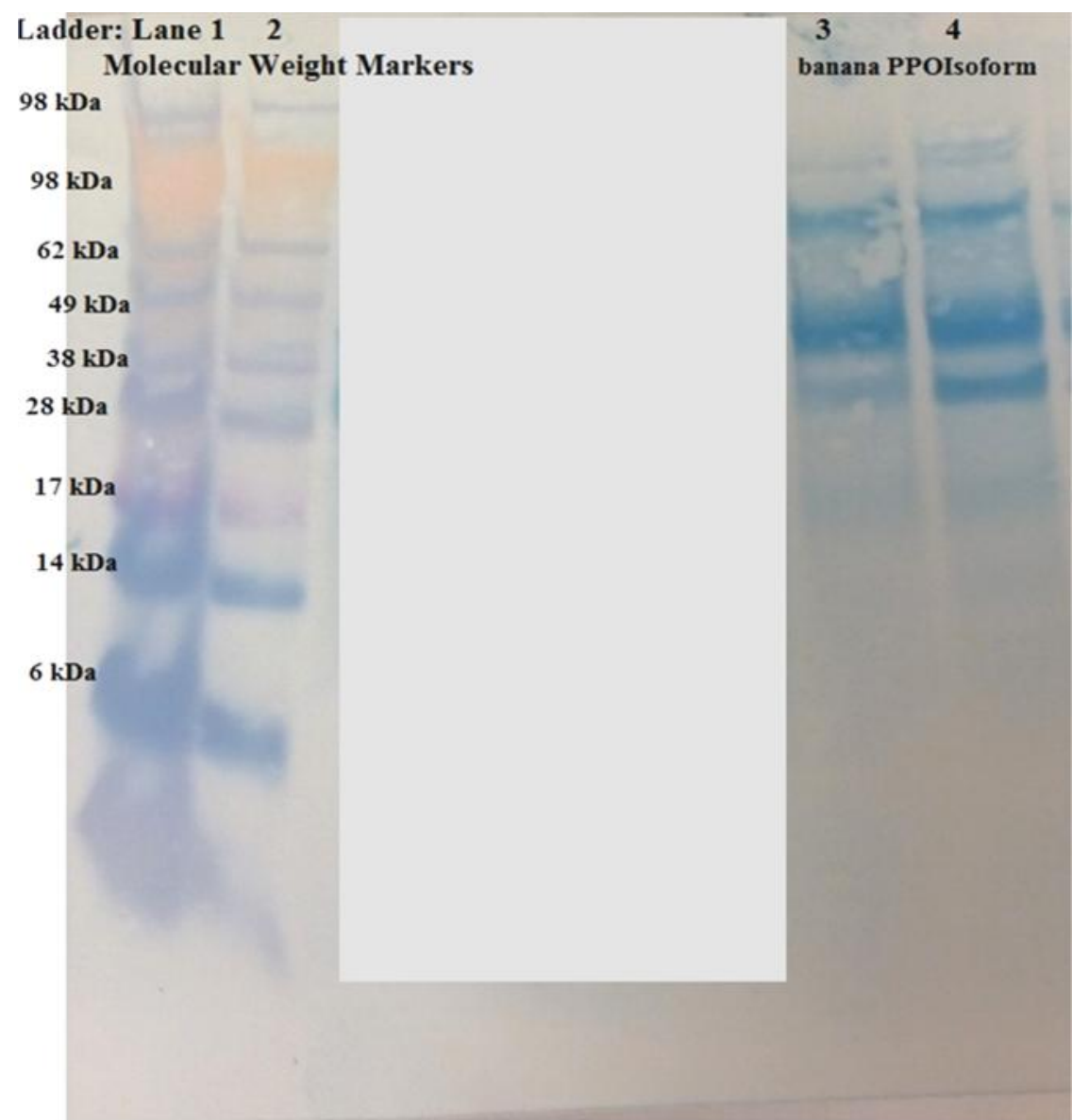

Figure 3. SDS PAGE of banana fruit pulp PPO isoforms; Lane $1 \&$ 2: molecular weight markers (198, 98, 62, 49, 38, 28, 17, 14, 6 kDa)). Lane 3 \& 4: banana PPO isoforms (28, 45 and $65 \mathrm{kDa})$ molecular weight.

\section{Efiect of pH on PPO Activity of Banana}

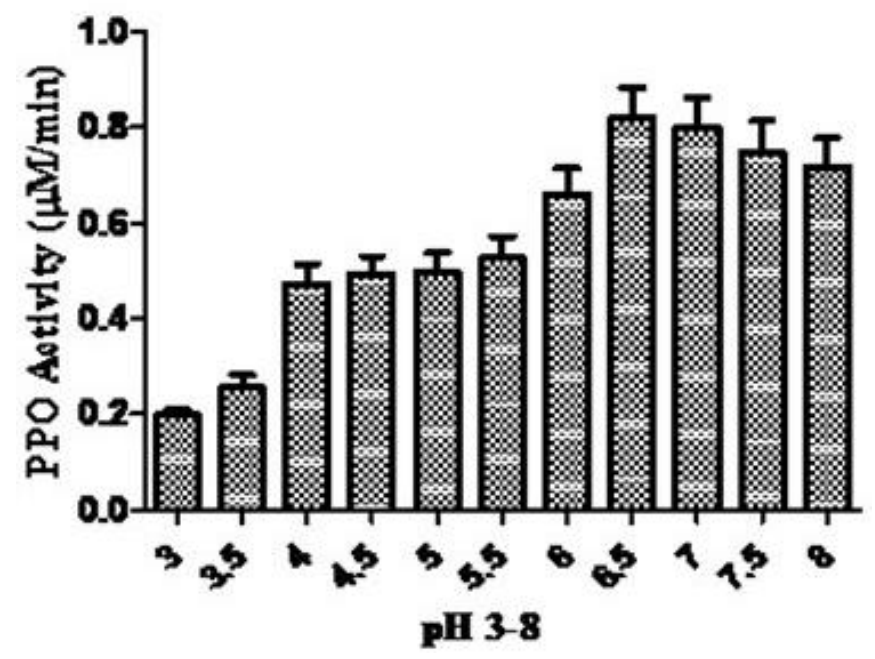

Figure 4. The pH optimization of PPO extracted from banana fruit pulp. Each bar plot represents mean value of the PPO activity at varying $\mathrm{pH}(3,3.5,4,4.5,5,5.5,6,6.5,7,7.5$ and 8$)$. Error bars are representation of standard error (SE) of the data. Phosphate buffer of varying $\mathrm{pH}$ is plotted on horizontal axis and PPO activity at different $\mathrm{pH}$ is plotted on vertical axis. The optimum PPO activity was observed at pH 6.5 and 7. 


\section{Efiect of Te=perature on PPO Activity of Banana}

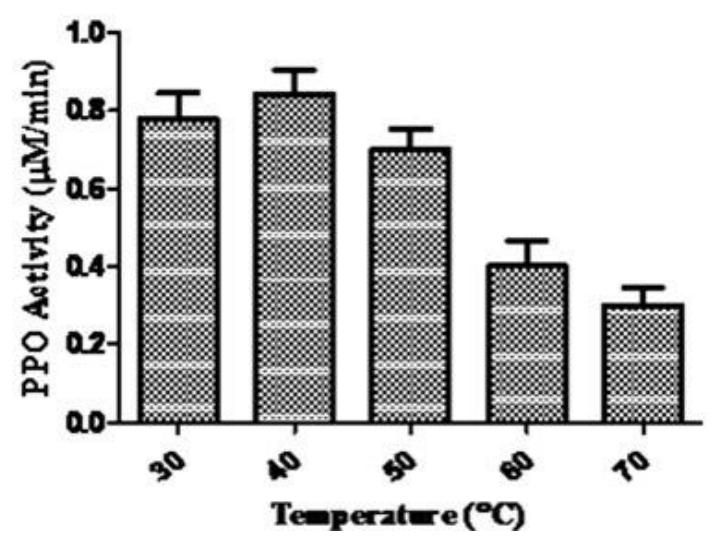

Figure 5. Temperature optimization of PPO extracted from banana fruit pulp. Each bar plot represents mean value of the $\mathrm{PPO}$ activity at varying temperatures $\left(30^{\circ} \mathrm{C}, 40^{\circ} \mathrm{C}, 50^{\circ} \mathrm{C}, 60^{\circ} \mathrm{C}\right.$ and $\left.70^{\circ} \mathrm{C}\right)$. Error bars are representation of standard error (SE) of the data. The temperature in ${ }^{\circ} \mathrm{C}$ is plotted on horizontal axis and PPO activity is plotted on vertical axis. The optimum PPO activity was observed at $30-40^{\circ} \mathrm{C}$ temperature.

Efiect of Metal Ions on PPO Activity of Banana

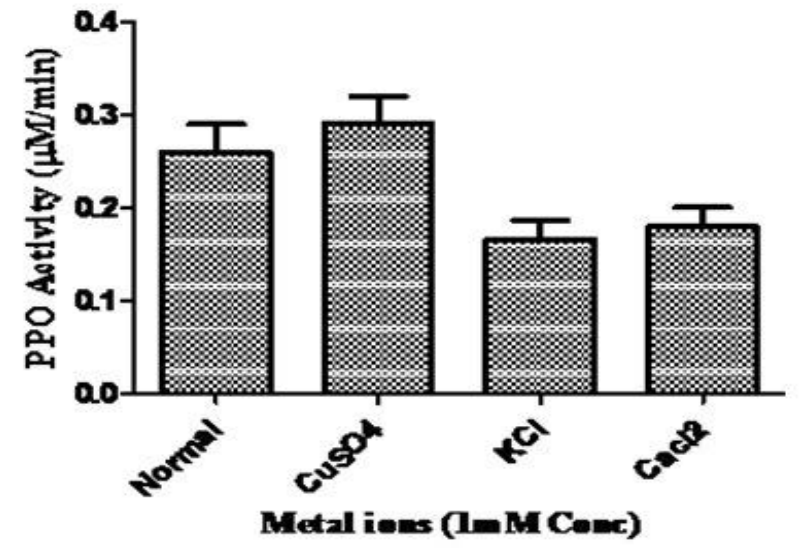

Figure 6. Effect of metal ions on PPO activity of banana fruit pulp. Each bar plot represents mean value of the PPO activity in the presence of metal ions. First bar is showing mean value of PPO activity in the absence of metal ions. Remaining three bar plots are showing PPO activity of banana fruit pulp in the presence of metal ions $\left(\mathrm{CuSO}_{4}, \mathrm{KCl}, \mathrm{CaCl}_{2}\right)$. Error bars are representation of standard error (SE) of the data. The metal ion presence $(1 \mathrm{mM}$ Conc) is plotted on horizontal axis and PPO activity of banana fruit pulp is plotted on vertical axis.

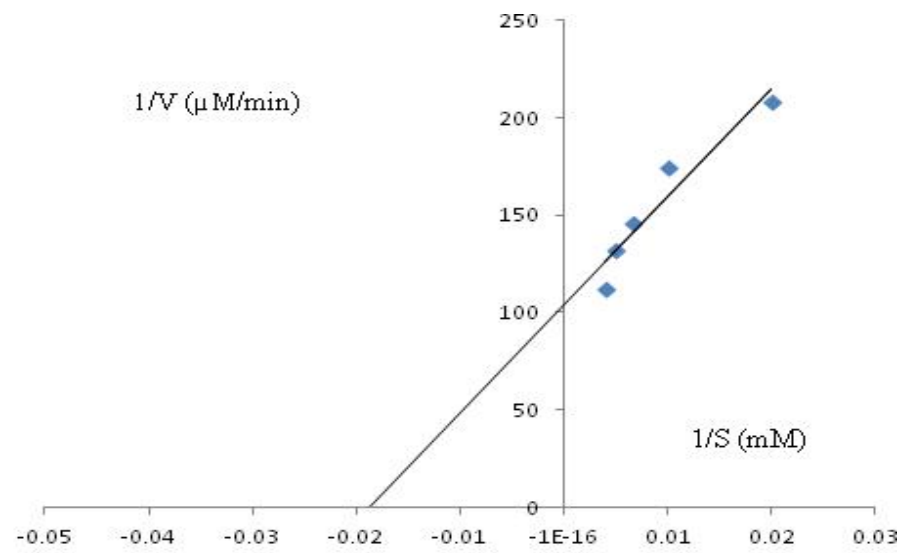

Figure 7. Lineweaver-burk Plot of banana fruit pulp PPO. 

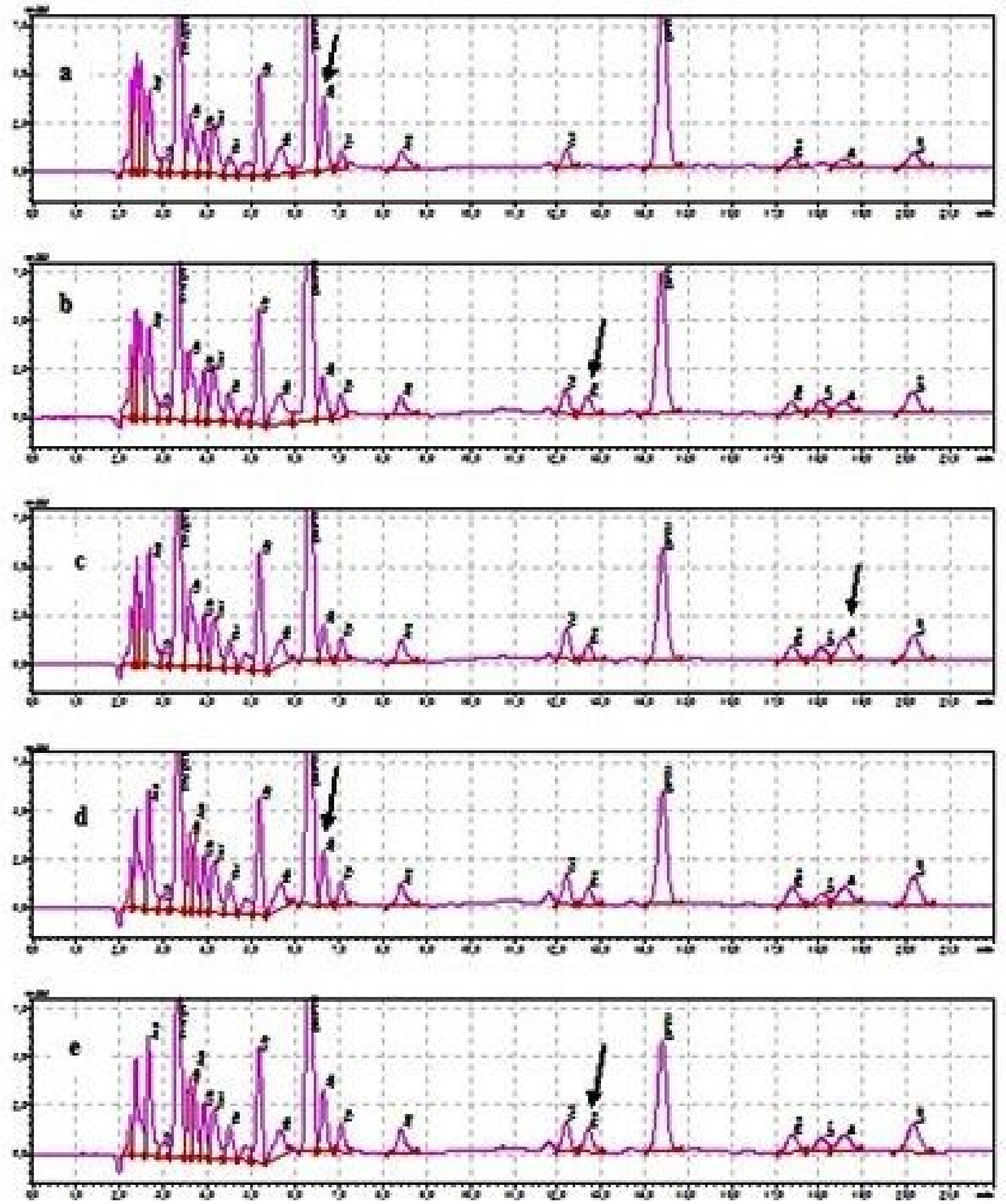

Figure 8 (A-E). Graphs showing N-terminal microsequencing of purified PPO isoform (45 kDa) from banana fruit pulp. (A) Graph showing Alanine residue. (B) Graph showing Proline residue. (C) Graph showing Isoleucine residue. (D) Graph showing Alanine residue. (E) Graph showing Proline residue. Arrow head symbol in the graphs are representing peaks of sequenced amino acid. 

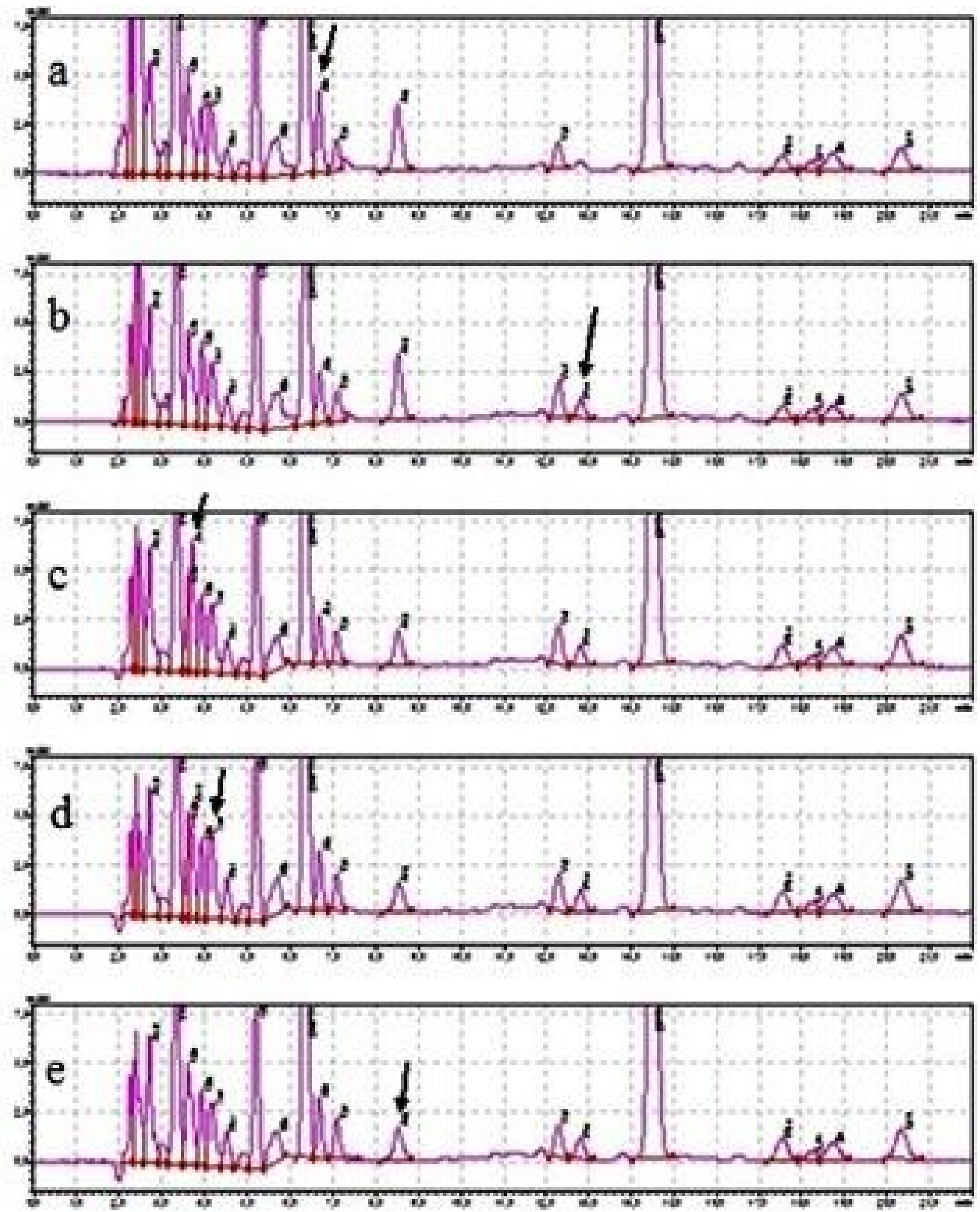

Figure 9 (A-E) Graphs showing N-terminal microsequencing of purified PPO isoform $(28 \mathrm{kDa})$ from banana fruit pulp. (A) Graph showing Alanine residue. (B) Graph showing Proline residue. (C) Graph showing Asparagine residue. (D) Graph showing Serine residue. (E) Graph showing Tyrosine residue. Arrow head symbol in the graphs are indicating peaks of the sequenced amino acid. 
A

AHH92831.1 MVSLFRATL.RLSLSFPSISASNSASFACAFHFSYPDRRRHAHPKISCR.ASDEHEMTANU ACl65307.1

B4

B3

AHH92831. KLDRRDVLVGLGGLOGAAALGIDSRALGTPIQAPDLTKCGPADLFTGATFNACCPYFP ACJ65307.1

B4

B3

AHH92831 1 DKRIDFKRPFNSSPLRVRPAAHLVDD YLXYKKAVELMRALPADDPRANMQQANVHCA AC765307.1

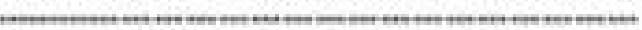

B4

B3

AHH92831: YCDGAYDQIGFPUELQVHESWLFFPWHRFYZYFHERILGKLIGDDTFALPFWNWDAPGG ACJ65307.1

B4

B3

AHH92831.1 MRLPSTYADPSSSLPKFRDAKHQPPVLVDLD NNGTDPSFTDAEQDQNLKMMYRQVISN ACJ65307.1

B4

B3

AHH928311 GKTPLLFLGSAYRAGDNPNPGAGSLENTPGPVHGWTGDRSCPNIEDMGEFYSAGRDPIF ACt65307.1

B4

B3

AHH92831.1 FAHHSNVDRMWYLWKKL.GGKHQDFNDWDLNTTFLFYDENADLVKVTL.KDCLQPEWLRYD

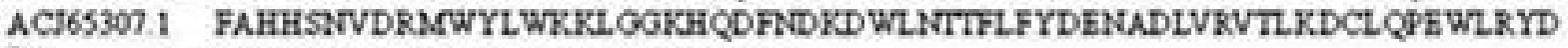
B4

B3

AHH92831.1 YQDVEIPWLKTRPTPRALKAQKTAAKTLKATAETPFPVTLQSAVSTTVRRPTVSRSGKER AC165307.1 YQDVEIPWLKTRPTPKALKAQKTAAKTLRATAETPFPVTLQSAVSTTVRRPKVSRSGKEK

B4

B3 APNSTAPLAP

\section{B}

\section{AHH92831.1 EEEEEVLIVEGIEFDRD YFIKFDVFVNATEGEGIPGASEFA GSFVNVPHKHKHSKKERK ACJ65307.1 EAEDESLA - \\ B4 \\ B3}

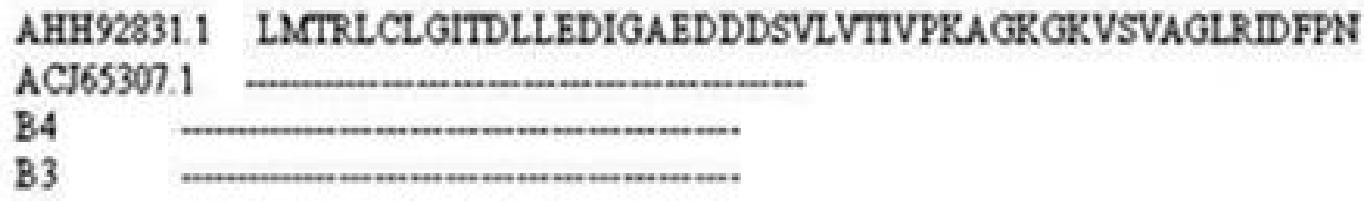

Figure 10 (A-B) Multiple sequence alignment of banana fruit pulp PPO isoform (28 kDa) with previously reported PPO sequences from other banana varieties. 


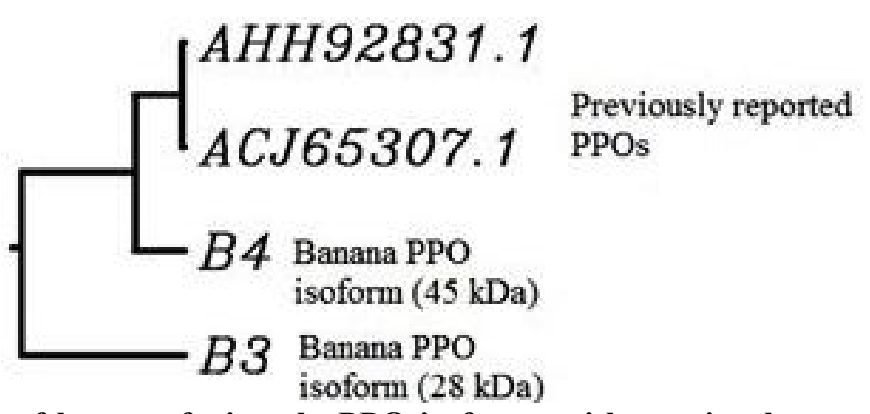

Figure 11. Phylogenetic tree of banana fruit pulp PPO isoforms with previously reported PPO sequenced from other banana varieties. In the above phylogenetic tree B3 (Banana isoform, $28 \mathrm{kDa}$ ) and B4 (Banana isoform, $45 \mathrm{kDa}$ ) are the PPO isoforms purified from banana apple fruit pulp in the current study. Diagram is showing relationship of B3 and B4 PPO isofoms with sequenced banana PPO (Accession ACJ65307.1 and AHH92831.1) available in databases.

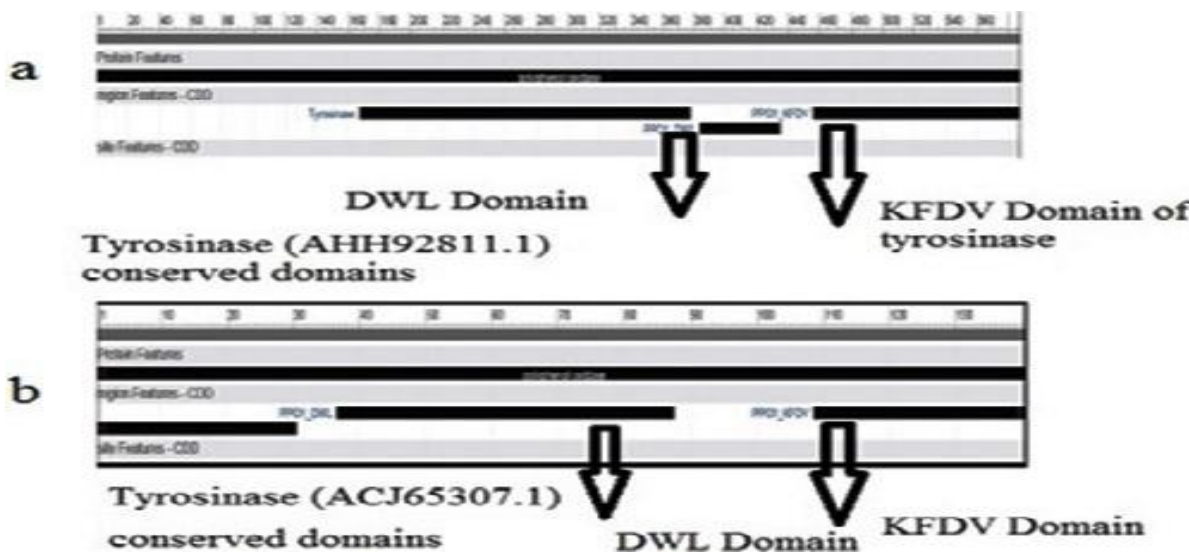

Figure 12 (A-B) Multiple sequence alignment showed sequence homology of purified and sequenced PPO isoforms from banana fruit pulp in this study with PPOs having accession no; ACJ65307.1 and AHH92831.1. (A) Tyrosinase (AHH92831.1) Conserved Domains. (B) Tyrosinase (ACJ65307.1) Conserved Domains.
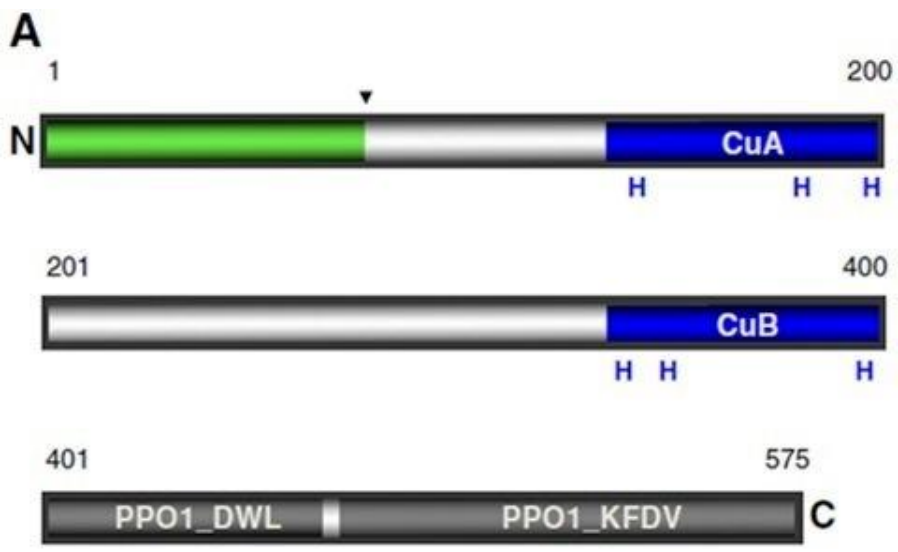

Figure 13. Schematic diagram of PPO domains and conserved residues. Typical PPOs contain N-terminal transit peptide (green), which is cleaved at an Alanine motif. The Conserved $\mathrm{CuA}$ and $\mathrm{CuB}$ domains are shown in Blue, the C-terminal domain in Grey.

\section{DISCUSSION}

Our findings showed PPO isoforms with maximum PPO activity from banana fruit pulp. The table
1 shows stepwise purification result of polyphenol oxidase. Total protein concentration was determined to be $0.635 \mathrm{mg} / \mathrm{mL}$, total yield $(30 \%)$ and purification folds (4.04) were also found. High yield purification of PPO 
isoforms with maximum PPO activity was observed in the present study. In line with previous studies the reported study on banana showed maximum PPO activity $(740 \mathrm{U} / 2 \mathrm{~mL})$ after fast protein liquid chromatography (Chaisakdanugull and Theerakulkait, 2009). One another study on banana (Musa acuminate Grande naine) showed maximum PPO activity using sodium phosphate buffer (Wuyts et al. 2006).

A further novel finding of the present study was the detection of three active bands $(28,45 \mathrm{kDa})$ on SDSPAGE. This result was then compared with previous studies where the PPO existence in number of isoforms was studied in different plants and reported studies on banana PPOs showed different number of PPO isoforms that might be possible due to developmental stages, artifact formation during purification, conditions for storage and native enzyme modifications (Harel et al. 1968; Lerner et al. 1972; Cash et al. 1976; Lieberei et al. 1978; Meyer et al. 1980). The covalent interactions between catalytically produced quinones and PPO protein could produce artifacts having different molecular weights and isoelectric points than the native one (Interesse et al. 1984; Kowalski et al. 1992). Modifications of PPO could also occur by the covalent attachment of glycosides to PPO (Flurkey et al. 1980; Raffert et al. 1995). The process of multiplicity was also described by the activation of the enzymes latent forms in few cases. Changes in the enzymes tertiary structure as a result of detergents and denaturing agents during different isolation methods used may also accounts for the multiplicity process of PPO enzyme. The existence of PPO in multiple isoforms may also be the result of differential expression of several different gene family members (Boss et al. 1995). Electrophoresis pattern of gel with showed three isenzymes of PPO (Chaisakdanugull and Theerakulkait, 2009). SDS-PAGE showed nine PPO isoenzymes in the interior of banana pulp (Montgomery et al. 1975). PPO isoforms migrated as three intense and 1 weak bands after activity staining with substrate (Catechol), while 4 bands stained with coomassie brillant R-250 during native PAGE. In Alanya banana five bands $(105 \mathrm{kDa}, 52 \mathrm{kDa}, 26 \mathrm{kDa}, 18 \mathrm{kDa}$ and $15 \mathrm{kDa}$ ) of PPO was observed by SDS-PAGE (Karakus and Pekyardimci, 2009).

The maximum PPO activity in the present study was observed at $\mathrm{pH} 6.5$ and 7. By comparing the result with reported studies on bananas, the optimum activity of polyphenol oxidase at pH 7 (Montgomery et al. 1975; Karakus and Pekyardimci 2009; Chaisakdanugull and Theerakulkait, 2009) was observed. The maximum PPO activity at $6.5 \mathrm{pH}$ was also investigated in another study (Chong et al. 2011).

The maximum PPO activity from banana fruit pulp was observed at $30-40^{\circ} \mathrm{C}$. A similar pattern of result was obtained in a previously reported study and it was found that the best temperature for $\mathrm{PPO}$ was $30^{\circ} \mathrm{C}$ (Yang et al. 2000). Previously, it has been studied that the maximum activity of banana $\mathrm{PPO}$ was found at $25-30^{\circ} \mathrm{C}$ with catechol substrate (Karakus and Pekyardimci, 2009). The optimum temperature $\left(30^{\circ} \mathrm{C}\right)$ was also observed in a study (Chong et al. 2011).

The PPO activity was increased in the presence of copper ions. The increased in PPO activity was observed in the reported study (Guray, 2009). The increase in PPO activity was also observed after the addition of copper ions in apple fruit (Aydin et al. 2015). The $\mathrm{K}_{\mathrm{m}}$ and $\mathrm{V}_{\max }$ for banana PPO $(53.33 \mathrm{mM}, 9.15$ $\mu \mathrm{M} / \mathrm{min}$ ) were calculated in the given study. $\mathrm{K}_{\mathrm{m}}$ in case of $20 \mathrm{mM}$ catechol substrate was $15.95 \mathrm{mM}$ in Alanya banana (Musa cavendishi) (Karakus and Pekyardimci, 2009). Compared with other fruits the $K_{m}$ and $V_{\max }$ for apple fruit pulp PPO were found to be $74.21 \mathrm{mM}$ and $4.45 \mu \mathrm{M} / \mathrm{min}$. $K_{m}$ of PPOs of different fruits vary as $K_{m}$ from Victoria grape $(52.6 \mathrm{mM})$, cocord grape $(67 \mathrm{mM})$, apple $(230 \mathrm{mM})$ and red delicious apple $(220 \mathrm{mM})$ (Rapeanu et al. 2006; Rocha et al. 2011; Satjawatcharaphong et al. 1983). N-terminal sequences of both purified PPO isoforms showed alanine as first amino acid residue. Alanine was the first amino acid appeared at $\mathrm{N}$-terminal region and it is reported that alanine residue appeared in the X-ray structure of PPO at the N-terminal region of the mature protein (Virador et al. 2010).

Conclusions: In the current study, three novel PPO isoforms $(65 \mathrm{kDa}, 45 \mathrm{kDa}, 28 \mathrm{kDa})$ extracted from fresh banana fruits pulp were characterized. The maximum PPO activity for these PPO isoforms was found at $\mathrm{pH}$ 6.5. The most favorable temperature for PPO activity was found in a range of 30 to $40^{\circ} \mathrm{C}$ and highest activity was achieved at $40^{\circ} \mathrm{C}$. The $\mathrm{K}_{\mathrm{m}}(53.33 \mathrm{mM})$, and $\mathrm{V}_{\max }(9.15$ $\mu \mathrm{M} / \mathrm{mL}$ ) values using catechol substrate under optimal conditions were determined by means of LineweaverBurk plot. N-terminal microsequencing of the purified PPO isoforms from banana fruit pulp was applied for the first time and showed APNST (28 kDa), and APIAP (45 $\mathrm{kDa}$ amino acid residues. Furthermore, the multiple sequence alignment by CLUSTALW showed these tryosinase isoforms are strongly related with banana PPO (AHH92831.1 and ACJ65307.1). The high yield of two novel PPO isoforms from banana fruit pulp is a breakthrough for their efficient use in biotechnological applications in medicine, environmental technology, textile industry, Pulp and paper industry and food industry as well as useful in future to predict crystallized structures. This approach can be further used to purify PPO isoforms to their crystalline structure. Further studies to predict the crystalline structure of these PPO isoforms and their genetics can be done to improve their activity.

Acknowledgements:The financial support from HEC through NRPU\#10406 and IRSIP funding to Ms. N. Sajjad is highly acknowledged. 


\section{REFERENCES}

Aydin, B., I. Gulcin, and S.H. Alwasel (2015). Purification and characterization of polyphenol oxidase from Hemsin apple. Int. J. food Proper. 18(12): 2735-2745.

Anaya-Esparza, L. M., R. M. Velázquez-Estrada, S. G. Sayago-Ayerdi, J. A. Sánchez-Burgos, M. V. Ramírez-Mares, M. D. L. García-Magaña, and E. Montalvo-González (2017). Effect of thermosonication on polyphenol oxidase inactivation and quality parameters of soursopnectar. LWT - Food Sci. and Technol. 75: 545-551.

Bravo, K. and E. Osorio (2016). Characterization of polyphenol oxidase from Cape gooseberry (Physalis peruviana L.) fruit. Food Chem. 197(Pt A): 185-190.

Boss, P. K., R. C. Gardner, B. Janssen, and G.S. Ross (1995). An apple polyphenol oxidase cDNA is up-regulated in wounded tissues. Plant Mol. Biol. 27: 429-433.

Constabel, C. P., Y. Lynn, J. J. Patton, and M. E. Christopher (2000). Polyphenol oxidase from hybrid poplar Cloning and expression in response to wounding and herbivory. Plant Physiol. 124: 285-295.

Claus, H. and H. Decker (2006). Bacterial tyrosinases. Systematic Appl. Microbiol. 29(1): 3-14.

Cao, X., C. Cai, Y. Wang, and X. Zheng (2017). The inactivation kinetics of polyphenol oxidase and peroxidase in bayberry juice during thermal and ultrasound treatments. Innov. Food Sci. and Emerging Technol. 45: 169-178. doi:10.1016/j.ifset.2017.09.018

Chaisakdanugull C. and C. Theerakulkait (2009). Partial purification and characterization of banana (Musa (AAA Group) Gros Michel) polyphenol oxidase. Food Sci. Technol. 44: 840-846.

Chong, L. C., L. H. Cheng, and A. A. Noor Aziz (2011). Properties of polyphenol oxidase obtained from Musa acuminata 6 balbisiana Colla cv. "Pisang Awak" pulp and peel. CyTA J. Food. 9: 25-30.

Cash, J. N., W.A. Sistrunk, and C. A. Stutte (1976). Characteristics of Concord grape polyphenoloxidase involved in juice color loss. Food Sci. 41: 1398-1402.

Flurkey, W. H. and J. J. Jen (1980). Purification of peach polyphenol oxidase in the presence of added protease inhibitors. J. Food Biochem. 4: 29-41.

Guray, M. Z. (2009). Partial purification and characterization of polyphenol oxidase from thermophilic bacillus sp. MS. thesis (Published). Grad. School of Engin. and Sci. Izmir Instit. of technology.
Guven, R. G., K. Guven, F. M. Bekler, O. Acer, H. Alkan, and M. Dogru (2017). Purification and characterization of polyphenol oxidase from purslane. Food Sci. Technol. 37: 356-362.

Hou, M. F., X. Y. Tang, W. D. Zhang, L. Liao, and H. F. Wan (2011). Degradation of pentachlorophenol by potato polyphenol oxidase. J. Agri. Food Chem. 59: 11456-11460.

Halaouli, S., M. Asther, J. C. Sigoillot, M. Hamdi, and A. Lomascolo (2006). Fungal tyrosinases: new prospects in molecular characteristics, bioengineering and biotechnological applications. J. Appl. Microbiol. 100 (2): 219232.

Harel E. and A. M. Mayer (1968). Interconversion of sub-units of catechol oxidase from apple chloroplasts. Phytochem. 7: 199-204.

Interesse, F. S., V. Alloggio, F. Lamparelli, and G. Avella (1984). Characterization of the oxidative enzymatic system of the phenolic compounds from Muscat grapes. Lebensm.-Wiss u-Technol. 17: 5-10.

Jesus, A., T. S. Leite, and M. Cristianini (2018). High isostatic pressure and thermal processing of açaí fruit (Euterpe oleracea Martius): Effect on pulp color and inactivation of peroxidase and polyphenol oxidase. Food Res. Int. 105: 853862.

Kong, K. H., M. P. Hong, S. S. Choi, Y.T Kim, and S. H. Cho (2000). Purification and characterization of a highly stable tyrosinase from Thermomicrobium roseum. Biotechnol. and Appl. Biochem. 31(2): 113-8.

Karakus, E. and S. Pekyardimci (2009). Purification and biochemical characterization of polyphenol oxidase from Alanya banana (Musa carevendishi). Asian J. Chem. 21: 3138-3150.

Kumar, V. B. A., T. C. K. Mohan, and K. Murugan (2008). Purification and kinetic characterization of polyphenol oxidase from Barbados cherry (Malpighia glabra L.) Food Chem. 11: 328-333.

Kowalski, S. P., N. T. Eannetta, A. T. Hirzel, and J. C. Steffens (1992). Purification and characterization of polyphenol oxidase from glandular trichomes of Solanum berthaultii. Plant Physiol. 100: 677-684.

Lerner, H. R., A. M. Mayer, and E. Harel (1972). Evidence for conformational changes in grape catechol oxidase. Phytochem. 11: 2415.

Lieberei, R. and B. Biehl (1978). Activation of latent phenolase from spinach chloroplasts by ageing and by frost. Phytochem. 17: 1427-1429.

Montgomery, M. W. and V. C. Sgarbieri (1975). Isoenzymes of banana polyphenol oxidase. Phytochem. 14: 1245-1249. 
Munoz-Pina, S., J. V. Ros-Lis, A. Argüelles, C. Coll, R. Martínez-Máñez, and A. Andrés (2018). Full inhibition of enzymatic browning in the presence of thiol-functionalised silica nanomaterial. Food Chem. 241; 199-205.

Mohammadi, M. and H. Kazemi (2002). Changes in peroxidase and polyphenol oxidase activities in susceptible and resistant wheat heads inoculated with Fusarium graminearum and induced resistance. Plant Sci. 162: 491-498.

Mahmood, W. A., S. S. Sultan, and S. R. Hamza (2009). Extraction and characterization of polyphenol oxidase from apricot, apple, eggplant and potato. Mesopotamia J. Agric. 27(4): 1815-316.

Meyer H. and B. Biehl (1980): Activities and multiplicity of phenolase from spinach chloroplasts during leaf ageing. Phytochem. 19: 2267-2277.

Mayer A. M. (2006). Polyphenol oxidases in plants and fungi: going places? A review. Phytochem. 67: 2318-2331.

Nematpour, F. S., K. Haghbeen, M. K. Babaei, F. R. Jazii, O. Nouraeen, and M. B. Yancheshmeh (2008). The banana pulp polyphenol oxidase is a tyrosinase. Sci. Alert. 526-533.

Ono, E., M. Hatayama, Y. Isono, T. Soto, R. Watanabe, and K. Yonekura (2006). Localization of a flavonoid biosynthetic polyphenol oxidase in vacuoles. Plant J. 45: 133-143.

Polaina, J. and A. P. MacCabe (2007). Industrial enzymes: structure, function and applications. $1^{\text {st }}$ Ed. Netherlands. 461p.

Paul S., C. Bird, and S. Robinson (2001). Molecular cloning and characterization of banana fruit polyphenol oxidase. Planta. 213: 748-757.

Queiroz, C., M. L. M. Lopes, E. Fialho, and V. L. Valentemes (2008). Polyphenol oxidase: Characteristics and mechanisms of browning control. Food Review Inter. 4: 361-375.

Rocha, A. M. C. N. and A. M. M. B. Morais (2001). Characterization of polyphenoloxidase (PPO) extracted from jonagoredo apple. Food cont. 12: 85-90.

Rapeanu, G., A. V. Loey, C. Smout, and M. Hendrick (2006). Biochemical characterization and process stability of polyphenoloxidase extracted from victoria grape (Vitis vifera ssp. Sativa). Food Chem. 94: 253-261.

Raffert G. and W. H. Flurkey (1995). Carbohydrate associated with broad bean polyphenol oxidase is resistant to enzymatic and chemical deglycosylation. Phytochem. 38: 1355-1360.

Sikora, M. and Świeca, M. (2018). Effect of ascorbic acid postharvest treatment on enzymatic browning, phenolics and antioxidant capacity of stored mung bean sprouts. Food. Chem. 239: 11601166.

Siddiq, M. and K. D. Dolan (2017). Characterization of polyphenol oxidase from blueberry (Vaccinium corymbosum L.). Food Chem. 218: 216-220.

Simsek, S. and A. Yemenicioglu (2007). Partial purification and kinetic characterization of mushroom stem polyphenoloxidase and determination of its storage stability in different lyophilized forms. Process Biochem. 6, 943-950.

Satjawatcharaphong, C., K. S. Rymal, J. R. Dozier, and R. C. Smith (1983). Polyphenol oxidase system in Red Delicious apples. J. Food Sci. 48: 1879. 1880.

Sojo, M. M., E. Nunez-Delicado, F. Garcia-Carmona, and A. Sanchez-Ferrer (1998a). Partial purification of a banana polyphenol oxidase using Triton X114 and PEG 8000 for removal of polyphenols. J. Agri. and Food Chem. 46: 4924-4930.

Taranto, F., A. Pasqualone, G. Mangini, P. Tripodi, M. M. Miazzi, S. Pavan, and C. Montemurro (2017). Polyphenol Oxidases in Crops: Biochemical, Physiological and Genetic Aspects. Int. J. Mole. Sci. 18: 377.

Teng, J., Z. Gong, Y. Deng, L. Chen, Q. Li, Y. Shao, and W. Xiao (2017). Purification, characterization and enzymatic synthesis of theaflavins of polyphenol oxidase isozymes from tea leaf (Camellia sinensis). LWT-Food Sci. and Technol. 84: 263-270.

Virador, V. M., J. P. Reyes, A. Blanco, A. Mendiola, E. G. M. Smith, and A. N. Moren (2010). Cloning sequencing, purification, and crystal structure of Grenache (Vitis vinifera) polyphenol oxidase. J. Agri Food Chem. 58: 1189-1201.

Wuyts, N., D. D. Waele, and R. Swennen (2006). Extraction and partial characterization of polyphenol oxidase from banana (Musa acuminata Grande naine) roots. Plant Physiol. and Biochem. 44: 308-314.

Yang, C. P., S. Fujita, M. D. Ashrafuzzaman, N. Nakamura, and N. Hayashi (2000). Purification and characterization of polyphenol oxidase from banana (Musa sapientum L.) pulp. J. Agri. Food Chem. 48: 2732-2735. 